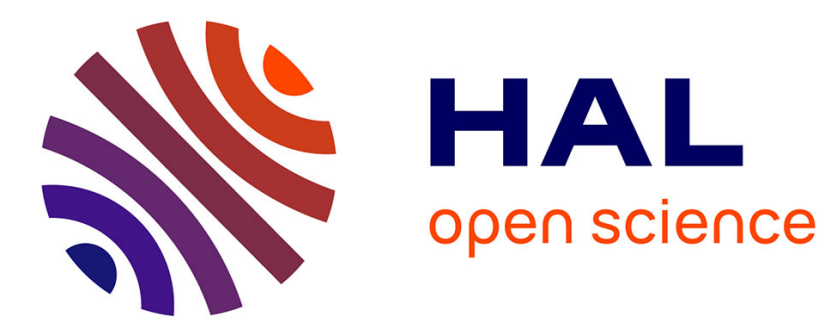

\title{
Study of the bending modes in circular quartz resonators
} Philippe Leclaire, Jozefien Goossens, Loïc Martinez, Nicolas Wilkie-Chancelier, Stéphane Serfaty, Christ Glorieux

\section{To cite this version:}

Philippe Leclaire, Jozefien Goossens, Loïc Martinez, Nicolas Wilkie-Chancelier, Stéphane Serfaty, et al. Study of the bending modes in circular quartz resonators. IEEE Transactions on Ultrasonics, Ferroelectrics and Frequency Control, 2006, 10.1109/TUFFC.2006.126 . hal-01324416

\section{HAL Id: hal-01324416 https://hal.science/hal-01324416}

Submitted on 1 Jun 2016

HAL is a multi-disciplinary open access archive for the deposit and dissemination of scientific research documents, whether they are published or not. The documents may come from teaching and research institutions in France or abroad, or from public or private research centers.
L'archive ouverte pluridisciplinaire HAL, est destinée au dépôt et à la diffusion de documents scientifiques de niveau recherche, publiés ou non, émanant des établissements d'enseignement et de recherche français ou étrangers, des laboratoires publics ou privés. 


\title{
Study of the Bending Modes in Circular Quartz Resonators
}

\author{
Philippe Leclaire, Jozefien Goossens, Loïc Martinez, Nicolas Wilkie-Chancelier, Stéphane Serfaty, \\ and Christ Glorieux
}

\begin{abstract}
An experimental and theoretical study of bending modes in a partially electroded circular piezoelectric quartz (AT-cut) with free edge is presented. The quartz is excited by a voltage pulse applied on the electrodes, and its surface is scanned by a laser vibrometer that measures the out-of-plane displacements. The classical theory of bending of thin disks is used to describe the flexural modes at frequencies lower than the first thickness shear resonance $(6 \mathrm{MHz})$. A fairly good agreement is found between experimental and theoretical results for the forced mode shapes and for the resonance frequencies. However, it appears that the two springs used to maintain the disk in position introduce extra clamping conditions. Several source shapes were studied, among which a collection of an arbitrary number of forces is particularly useful. The two-dimensional wavenumber representation shows the presence of anisotropy related to the crystallographic axes at higher frequencies, which is not predicted by the model. The experimental phase velocities are compared to those given by the classical theory of disks and to those of Lamb $A_{0}$ mode. This study confirms the correspondence at low frequencies between the $A_{0}$ mode and the bending eigenmodes of a disk with finite size.
\end{abstract}

\section{INTRODUCTION}

$\mathrm{P}$ IEZOELECTRIC quartz resonators are widely used as frequency-time delay control devices in numerous electronic appliances such as watches, cellular phones, television sets, or computers. In these applications, the highquality factor of quartz and the low-dependency of the resonance frequency to perturbing parameters result in an accurate control of the resonance frequency or time period. Quartz is anisotropic and, depending on the way it is cut, it also can be used in other applications as sensors for temperature, stress, or pressure for example. A review of various applications of homogeneous and composite quartz has been given by Benes et al. [1]. In one of these applications, the surface of an AT cut quartz is applied to a Newtonian liquid with a viscosity $\eta[2]$. The thickness shear resonance is used to generate a rapidly vanishing wave in the liquid with a typical viscous skin depth of a few micrometers. Thickness resonances of a quartz can be described by the equations of piezoelectricity. The boundary conditions are applied to the governing equation of piezoelectricity and, for a plate of infinite lateral extent, the problem can be treated as one dimensional [3]. Equations, including the lateral dependency, also have been established and used to study plates with varying thickness such as the beveled plate with tapered surface [4]. Détaint et al. [5] studied the effect of the electrode shape on the forced resonance modes of planes and corrugated resonators.
In this article, we present a study of the bending modes that occur at low frequencies (as opposed to the higher frequency thickness modes) in an AT-cut quartz disk used in shear thickness mode for monitoring the liquid-solid phase transition in gels [6]. It is shown experimentally from laser Doppler surface scanning experiments that these bending modes are efficiently excited. The normal and forced modes are studied theoretically, and a comparison with the experimental results is carried out for a disk with a free edge. To the best of our knowledge, theoretical and experimental results on the forced bending vibrations of free disks are scarce. Transverse modes as well as thickness modes in piezoceramic disks were studied recently by Huang et al. [7] and Huang [8]. The approach adopted here is different from the one by these authors in that the classical theory of thin plates and disks is used [9]. The equations of piezoelectricity are not used, and the stresses induced by the electrodes are taken to be external forces. The electromechanical coupling responsible for a stiffening of the material is not considered because it was shown by Huang [8] to be small.

The main benefit of the approach proposed here is that simpler equations are involved, that the effect of the source can be studied more easily, and that many source configurations can be modeled and tested. Different sources are included in the model, including a point force located arbitrarily on the disk, a circular line source, a constant pressure spread over a circular region, and a collection of an arbitrary number of forces, which is very convenient to model any source shape. The forced motion corresponding to the appropriate source is calculated as a function of frequency and compared with the experimental results. Other original aspects developed are the connection between the bending modes and the Lamb first antisymmetrical mode $\left(A_{0}\right)$ as well as the transient aspect of the wave propagation from the electrodes.

\section{Bending Modes of a Circular Plate with FREE EDGE}

\section{A. Normal Modes}

The normal modes (following the terminology in [9]) correspond to the "natural" vibration modes associated 
with particular shapes of the disk surface. The normal modes can occur only at certain frequencies determined by the disk properties and edge conditions, the natural frequencies. The normal modes also can be called the eigenmodes for the reason that they form a basis of functions upon which any forced vibration at a frequency imposed by the source can be decomposed.

A disk of thickness $h$, radius $a$, density $\rho$, Young's modulus $E$, and Poisson ratio $\nu$ is considered. The classical plate equation is [9]:

$$
D \nabla^{4} w+h \rho \partial_{t}^{2} w=0
$$

where $w$ is the lateral displacement of the disk and $D$ is the bending stiffness, given by:

$$
D=\frac{E h^{3}}{12\left(1-\nu^{2}\right)} \text {. }
$$

In polar system of coordinates $(r, \theta)$, solutions of the form:

$$
w=W(r, \theta) e^{i \omega t}
$$

where $\omega$ is the angular frequency are admissible, and the plate equation can be written:

$$
\left(\nabla^{2}-\gamma^{2}\right)\left(\nabla^{2}+\gamma^{2}\right) W=0
$$

with:

$$
\gamma^{2}=\omega \sqrt{\frac{h \rho}{D}}
$$

This leads to the following possible general solutions for the plate equation:

$$
W={ }_{\sin }^{\cos }(n \theta) J_{n}(\gamma r) \text { or } W={ }_{\sin }^{\cos }(n \theta) I_{N}(\gamma r),
$$

where $J_{n}$ is the Bessel function of the first kind and $I_{n}$ is the modified Bessel function of the first kind:

$$
I_{n}=i^{-n} J_{n}
$$

The general solutions for the bending of a disk are of the form:

$$
W={ }_{\sin }^{\cos }(n \theta)\left[A J_{n}(\gamma r)+B I_{n}(\gamma r)\right]
$$

where $A$ and $B$ are constants to be determined from the boundary conditions.

The case of a disk with a clamped edge was treated by Morse and Ingard [9]. For a plate with a free edge, the boundary conditions are such that the radial bending moment and Kelvin-Kirchoff shear [10], [11] are zero at the edge of the disk at $r=a$. Using the notations of Gabrielson $[10]$ :

$$
A M_{n 1}(\gamma a)+B M_{n 2}(\gamma a)=0
$$

and:

$$
A V_{n 1}(\gamma a)+B V_{n 2}(\gamma a)=0
$$

with:

$$
\begin{aligned}
M_{n 1}(\gamma a)= & \left(\frac{n(n-1)(1-\nu)}{(\gamma a)^{2}}-1\right) J_{n}(\gamma a) \\
& +\frac{(1-\nu)}{\gamma a} J_{n+1}(\gamma a), \\
M_{n 2}(\gamma a)= & \left(\frac{n(n-1)(1-\nu)}{(\gamma a)^{2}}+1\right) I_{n}(\gamma a) \\
& -\frac{(1-\nu)}{\gamma a} I_{n+1}(\gamma a), \\
V_{n 1}(\gamma a)= & \frac{n}{\gamma a}\left(\frac{n(n-1)(1-\nu)}{(\gamma a)^{2}}+1\right) J_{n}(\gamma a) \\
& -\left(\frac{n^{2}}{\gamma a} \frac{(1-\nu)}{\gamma a}+1\right) J_{n+1}(\gamma a), \\
V_{n 2}(\gamma a)= & \frac{n}{\gamma a}\left(\frac{n(n-1)(1-\nu)}{(\gamma a)^{2}}-1\right) I_{n}(\gamma a) \\
& +\left(\frac{n^{2}}{\gamma a} \frac{(1-\nu)}{\gamma a}-1\right) I_{n+1}(\gamma a) .
\end{aligned}
$$

The roots of the determinant:

$$
\left|\begin{array}{cc}
M_{n 1} & M_{n 2} \\
V_{n 1} & V_{n 2}
\end{array}\right|=0
$$

impose the allowed values of $\gamma$. For a given value of $n$ corresponding to the number of nodal diameters $(n=$ $0,1,2, \ldots)$, the roots of the determinant provide $m$ values of allowed $\gamma, m$ corresponding to the number of nodal circles $(m=1,2, \ldots)$. The parameter $\gamma$ becomes $\gamma_{n m}$ and the frequencies of the normal modes are given by:

$$
\omega_{n m}=\gamma_{n m}^{2} \sqrt{\frac{D}{h \rho}}
$$

Eq. (11) corresponds to the dispersion relation between the frequencies and the spatial wavenumbers of the allowed modes. The amplitudes of the normal modes can be determined from either $(9 \mathrm{a})$ or $(9 \mathrm{~b})$ and from normalizing one of the constants, say $A=1$. $A$ and $B$ become $A_{n m}$ and $B_{n m}$, respectively, and:

$$
B_{n m}=-A_{n m} \frac{M_{n 1}\left(\gamma_{n m} a\right)}{M_{n 2}\left(\gamma_{n m} a\right)}=-A_{n m} \frac{V_{n 1}\left(\gamma_{n m} a\right)}{V_{n 2}\left(\gamma_{n m} a\right)} .
$$

The frequencies and the mode shapes of the normal modes are given by (8), (12), and (13). The squared velocity of the calculated normal modes of the free disk between 0 and $100 \mathrm{kHz}$ are shown in Fig. 1. The quartz used is anisotropic and belongs to the trigonal class of symmetry. However, it was considered as isotropic in the model, and the values of the Young's modulus and of the Poisson ratio were taken such that a good fit was obtained between experimental results and the model, which in its present form 


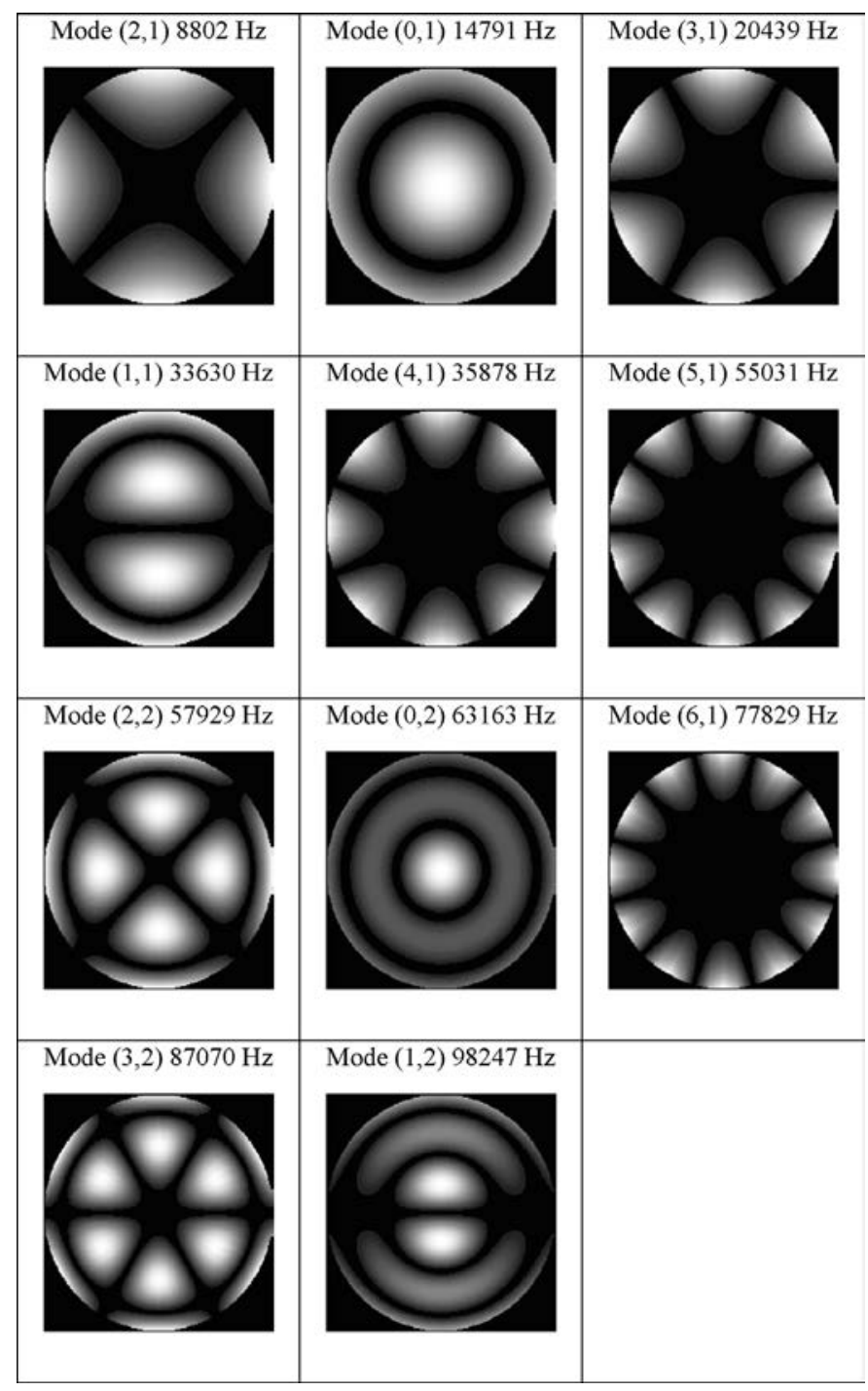

Fig. 1. The bending normal modes of a disk with a free edge in a frequency range between 0 and $100 \mathrm{kHz}$.

does not include several elastic coefficients. The properties used for the calculation were the following: a density of $\rho=2650 \mathrm{~kg} / \mathrm{m}^{3}$, a Young's modulus of $E=8.8 \times 10^{10} \mathrm{~Pa}$, and a Poisson ratio of 0.3. The value of Young's modulus of the crystal studied is between $9.72 \times 10^{10} \mathrm{~Pa}$ (crystal with crystallographic axis parallel to the $z$-axis) and $7.65 \times 10^{10} \mathrm{~Pa}$ (crystal with crystallographic axis perpendicular to the $z$-axis). The crystal used is cut at an angle of $35.25^{\circ}$ about the $z$ axis. It will be seen that, although a model for isotropic material is used, a fairly good match will be found between experimental and theoretical results.

\section{B. Forced Motion}

Forced motions are modeled by including a source term in the equation of motion, which becomes:

$$
D \nabla^{4} w+h \rho \partial_{t}^{2} w=s(r, \theta, t),
$$

where $s(r, \theta, t)$ has the dimensions of a pressure and is of the form:

$$
s(r, \theta, t)=S_{0}(r, \theta) e^{i \omega t} .
$$

The solutions of (14) are written as double infinite series:

$$
W(r, \theta)=\sum_{n=0}^{\infty} \sum_{m=1}^{\infty} W_{n m}^{e} R_{m n}(r) \cos (n \theta)
$$

or

$$
W(r, \theta)=\sum_{n=0}^{\infty} \sum_{m=1}^{\infty} W_{n m}^{o} R_{m n}(r) \sin (n \theta),
$$

where $W_{n m}^{e}$ and $W_{n m}^{o}$ are the amplitude coefficients of even and odd, forced modes and with:

$$
R_{n m}(r)=A_{n m} J_{n}\left(\gamma_{n m} r\right)+B_{n m} I_{n}\left(\gamma_{n m} r\right) .
$$

Inserting these series in the plate equation (14), multiplying each term by $R_{p q}(r) \cos (p \theta)$ with $p, q$ integers and integrating each term over the disk surface yields (18) (see next page) for the solution in $\cos (n \theta)$, where use has been made of the fact that, in polar coordinates, the biharmonic operator is:

$$
\nabla^{4} \equiv \gamma_{n m}^{4}
$$

Interchanging the integral and summation operators and making use of the orthogonality relations yield the amplitude coefficients:

$$
W_{n m}^{e}=\frac{\int_{0}^{2 \pi} \int_{0}^{a} S_{0}(r, \theta) R_{m n}(r) \cos (n \theta) r d r d \theta}{\left(D \gamma_{n m}^{4}-\rho h \omega^{2}\right) \int_{0}^{a} R_{n m}^{2}(r) r d r \int_{0}^{2 \pi} \cos ^{2}(n \theta) d \theta},
$$

and a similar expression for $W_{n m}^{o}$ where $\sin (n \theta)$ is involved instead of $\cos (n \theta)$. The orthogonality requirement is such that the terms with nonidentical indices $n, p$ and $m, q$ are zero. The forced solutions of the disk then are given by:

$$
\begin{aligned}
& w(r, \theta, t)= \\
& \left(\sum_{n=0}^{\infty} \sum_{m=1}^{\infty} W_{n m}^{e} R_{m n}(r) \cos (n \theta)+W_{n m}^{o} R_{m n}(r) \sin (n \theta)\right) e^{i \omega t}
\end{aligned}
$$

\section{Modeling the Source}

In this study, the stresses induced by the electrodes are taken to be external forces that are decoupled from the other stresses in the disk. This is possible because the electromechanical coupling responsible for a stiffening of the material is fairly small [8]. Also, the electrode thickness $(500 \mathrm{~nm})$ can be considered to be acoustically negligible. We have studied several source configurations from the general expressions of $W_{n m}^{e}$ and $W_{n m}^{o}$. 


$$
\begin{aligned}
\int_{0}^{2 \pi} \int_{0}^{a} \sum_{n=0}^{\infty} \sum_{m=1}^{\infty}\left(D \gamma_{n m}^{4}-\rho h \omega^{2}\right) W_{n m}^{e} R_{n m}(r) \cos (n \theta) R_{p q}(r) \cos (p \theta) r d r d \theta= \\
\int_{0}^{2 \pi} \int_{0}^{a} \sum_{n=0}^{\infty} \sum_{m=1}^{\infty} S_{0}(r, \theta) R_{p q}(r) \cos (p \theta) r d r d \theta,
\end{aligned}
$$

1. Point Force: For a point force $F_{0}$ located at $\left(r_{0}, \theta_{0}\right)$, the source term can be written as:

$$
S_{0}(r, \theta)=F_{0} \frac{\delta\left(r-r_{0}\right) \delta\left(\theta-\theta_{0}\right)}{2 \pi r} .
$$

$F_{0}$ has the dimensions of a force, $\delta\left(r-r_{0}\right)$ is expressed in $m^{-1}$ and $S_{0}$ is a pressure. From the property of the delta function:

$$
W_{n m}^{e}=\frac{F_{0} R_{m n}\left(r_{0}\right) \cos \left(n \theta_{0}\right)}{\left(D \gamma_{n m}^{4}-\rho h \omega^{2}\right) \int_{0}^{a} R_{n m}^{2}(r) r d r \int_{0}^{2 \pi} \cos ^{2}(n \theta) d \theta}
$$

2. Line Source: We consider the example of a line source consisting of a constant force applied on a concentric circle of radius $r_{0}$ :

$$
S_{0}(r, \theta)=F_{0} \frac{\delta\left(r-r_{0}\right)}{2 \pi r} \forall \theta .
$$

The symmetry of this source imposes the absence of angular modes, i.e., $n=0$; (19) then is simplified to:

$$
W_{0 m}^{e}=F_{0} \frac{R_{0 m}\left(r_{0}\right)}{2 \pi\left(D \gamma_{0 m}^{4}-\rho h \omega^{2}\right) \int_{0}^{a} R_{0 m}^{2}(r) r d r}
$$

3. Uniform Pressure: For a constant pressure $P_{0}$ on a disk of radius $r_{0}$ (smaller than the quartz radius):

$$
S_{0}(r, \theta)=P_{0} \text { for } r \leq r_{0} \text { and } \forall \theta
$$

Here too the symmetry imposes $n=0$ and:

$$
W_{0 m}^{e}=P_{0} \frac{\int_{0}^{r_{0}} R_{0 m}(r) r d r}{\left(D \gamma_{0 m}^{4}-\rho h \omega^{2}\right) \int_{0}^{a} R_{0 m}^{2}(r) r d r} .
$$

4. Collection of Forces: A collection of several forces can be simply modeled by adding their individual contributions. Anticipating the experimental configuration, a series of 20 forces oriented upward on the horizontal disk (Fig. 2), 20 forces oriented downward, spread over a circle of radius $r_{0}$ and with amplitudes varying sinusoidally

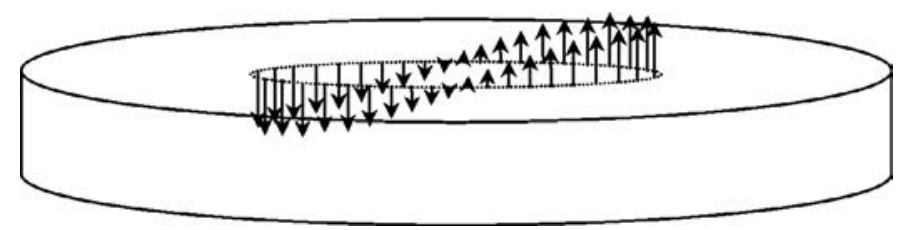

Fig. 2. Distribution of 40 forces on a circle of radius $r_{0}$ with sinusoidally varying amplitude starting from an angle of $-90^{\circ}$ (in this example).

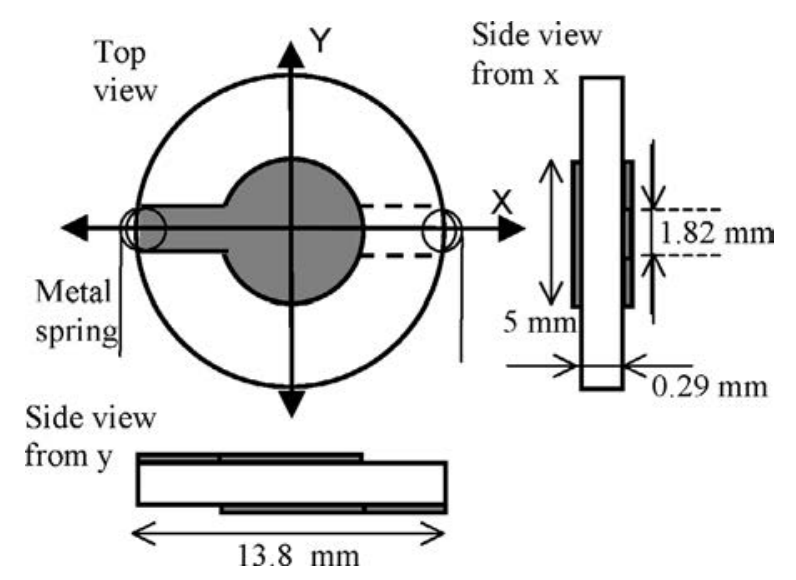

Fig. 3. Circular quartz resonator partially covered with electrodes.

starting from an initial angle $\theta_{0}$ has been modeled. For a series of forces starting at an angle of 0 radian, the amplitude coefficients are given by (27) (see next page).

The collection of forces as a source is particularly useful because it can be represented by a matrix of experimental data. In this study, both the series of 40 forces given by (27) and a matrix of 80 by 80 forces obtained from experimental results will be tested in the comparison between experimental and theoretical results.

\section{Laser Doppler Surface Scanning Experiments on a Circular Quartz Resonator}

The sample studied was a partially electroded AT-cut quartz disk (the crystal was cut along the $x$ and $y$ crystal axes and at an angle of $35.25^{\circ}$ with the $z$ axis) of $13.8 \mathrm{~mm}$ diameter, $0.29 \mathrm{~mm}$ thickness designed to have a thicknessshear fundamental frequency at $6 \mathrm{MHz}$ and with a free edge (Fig. 3). The disk was maintained in position with the help of two soft metal springs in contact with the rect- 


$$
W_{n m}^{e}=F_{0} \frac{\sin \left(\frac{\pi}{20}\right) R_{m n}\left(r_{0}\right) \cos \left(n \frac{\pi}{20}\right)+\cdots+\sin \left(\frac{40 \pi}{20}\right) R_{m n}\left(r_{0}\right) \cos \left(n \frac{40 \pi}{20}\right)}{\left(D \gamma_{n m}^{4}-\rho h \omega^{2}\right) \int_{0}^{a} R_{n m}^{2}(r) r d r \int_{0}^{2 \pi} \cos ^{2}(n \theta) d \theta} .
$$

angular parts of the electrodes and ensuring the electrical connection of the electrodes. The metal springs were made of very thin steel wires so that the plate could be considered to have a free edge. The electrodes are deposited gold disks of 5 -mm diameter. The width of the connecting strip is $1.82 \mathrm{~mm}$.

The experimental approach consisted in scanning the surface of the quartz by the beam of the laser vibrometer while the transducer was excited by a pulse generator at a repetition rate of a few kilohertz. The probe laser beam scanning was achieved by mounting a mirror/lens arrangement on an $x y$-controller so that the focusing point could move on the surface. The spatial step was $0.125 \mathrm{~mm}$. In order to get a reflective surface for the probe beam, the disk was covered with an acoustically negligible, very thin (less than $0.5 \mu$ ) layer of chromium by use of an evaporator.

The cut-off frequency of the displacement detecting Polytec Laser Doppler Vibrometer (Polytec PI, S.A., Pantin, France) was $20 \mathrm{MHz}$. This was high enough to visualize the transient response of the transducer and the most important eigenmodes. During the signal acquisition, a compromise was made between collecting long signal traces (198 $\mu$ s duration), in order to track several periods of the low-frequency eigenmodes, and keeping a high sampling rate $(50 \mathrm{MHz})$, in order to resolve the fast initial transient response. This was made possible by averaging rather large signal traces of $10^{4}$ points. The scanned region was a rectangular area that included almost the whole surface of the quartz (40 by 54 positions, with $250 \mu \mathrm{m}$ steps).

\section{Effect of the Electrode Shape on the Propagation of Bending Waves in the Disk}

\section{A. Transient Behavior of the Bending Waves}

The initial transient response resulting from a pulse applied to the electrodes is shown in Fig. 4 in which two surface waves are generated at the edge of the electrode, propagating along the radii of the disk. One wave focuses to the center, then defocuses toward the edge. The other wave propagates toward the edge, is reflected, and focuses to the center. The transient analysis also shows the effect of the connecting strip on the generation of surface waves. Although displacements are excited all over the electrode region, mainly the propagating sharp wave features are visible. These short wave packets are excited where the stress-gradient is large, i.e., at the electrode edges. Note the phase opposition between the upper and lower parts of the quartz, with an axial symmetry along the central horizontal axis $x$. This is due to the opposite exciting shear stress gradient at the electrode edges. The group velocity of the wave packets can be determined from their time-offlight. The value obtained is close to the shear velocity of quartz $\left(3570 \mathrm{~m} / \mathrm{s}\right.$ for a shear modulus of $3.38 \times 10^{10} \mathrm{~Pa}$ and a density of $2650 \mathrm{~kg} / \mathrm{m}^{3}$ ).

\section{B. Modeling the Source from the Experimental Data on the Transient Response}

Our aim is to compare experimental and theoretical modes; so the calculation of the source-excited mode shapes requires a realistic modeling of the source (the experimental results on the modes and their comparison with theory are presented in the next section). Fig. 5(a) shows the initial deformation of the disk near the electrode obtained from the transient analysis of the previous section. In order to approach this shape, the source was first modeled as a collection of 40 forces on a circle of radius $r_{0}$ with sinusoidally varying amplitude starting from an angle of $0^{\circ}$ as described in Section II-C. The symmetry of this theoretical source is such that only the modes which are symmetrical about the horizontal axis are predicted. It also appears that the connecting strips of the electrodes play a role and have to be included. Both the connecting strips and the two clamping springs are responsible for the existence of a horizontal symmetry axis (see Fig. 3) that does not exist in [8]. Yet, even after having accounted for the connecting strips, the theoretical source could not predict all the experimental results on the forced modes, and more modes than predicted were detected. For this reason, it was decided to improve the modeling of the source, and the solution chosen was to use the matrix of forces of Fig. 5(b) as a collection of forces in the model. Fig. 5(b) shows a 80 by 80 matrix corresponding to the experimental level of deformation on and near the electrode. At first sight, the source imposes a symmetry about the horizontal axis. However, asymmetries in the deformation over the surface and experimental noise [the black and white spots in Fig. 5(b)] are responsible for the breaking of the symmetry and will result in the excitation of all the modes. Another cause of breaking of the symmetry is the anisotropy of the crystal, which is not included in the model.

\section{Comparison Between Experimental and Theoretical Modes}

In this experiment, the pulsed excitation according to the symmetry of the electrodes initiates a continuum of vibrations in a wide band of frequencies. Mainly vibrations with frequencies corresponding to eigenmodes are excited 

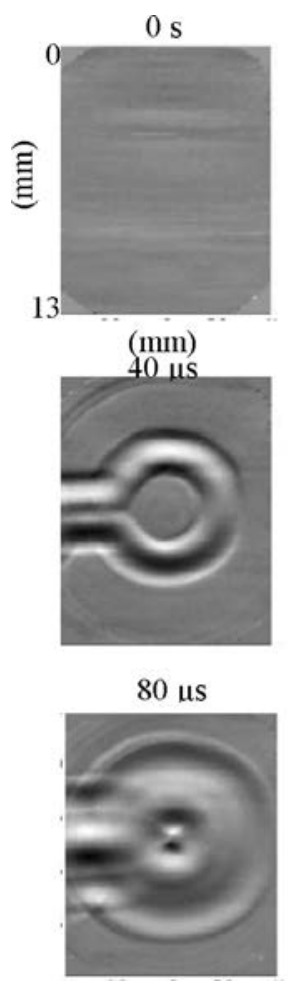

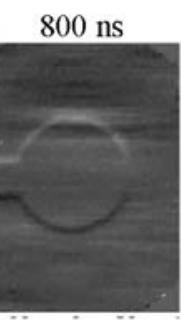

48 us

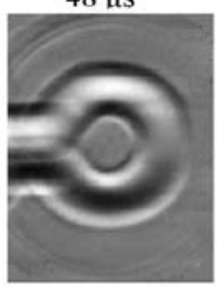

88 us

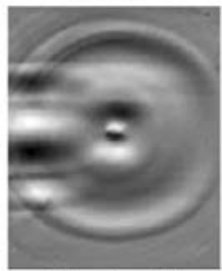

16 us

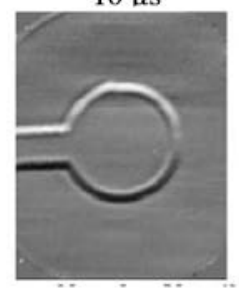

56 us

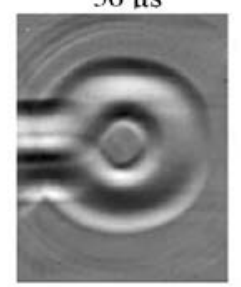

96 us

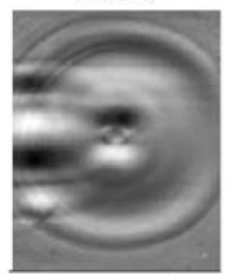

24 us

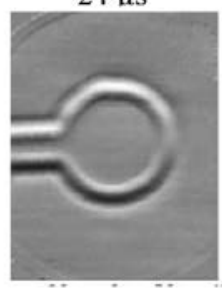

64 us

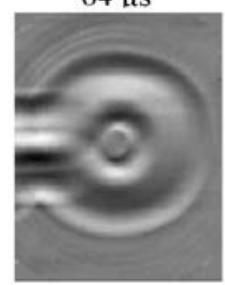

104 us

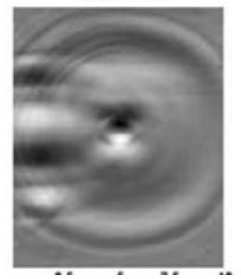

32 us

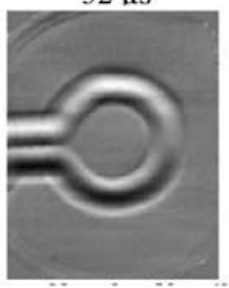

72 us

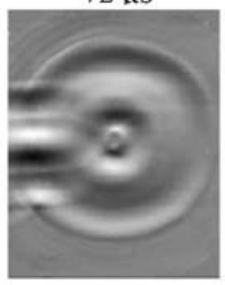

$112 \mu \mathrm{s}$

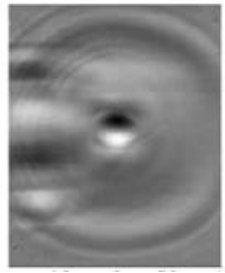

Fig. 4. Snapshots of the initial deformation of the disk surface. The dimensions of the disk are shown in the first image.

a)

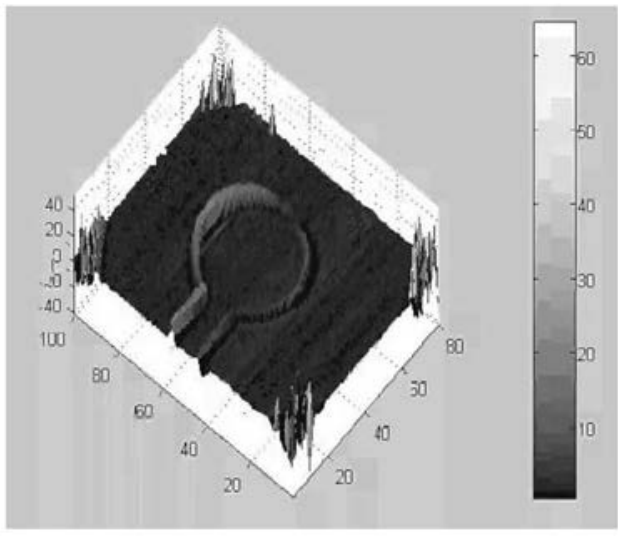

b)

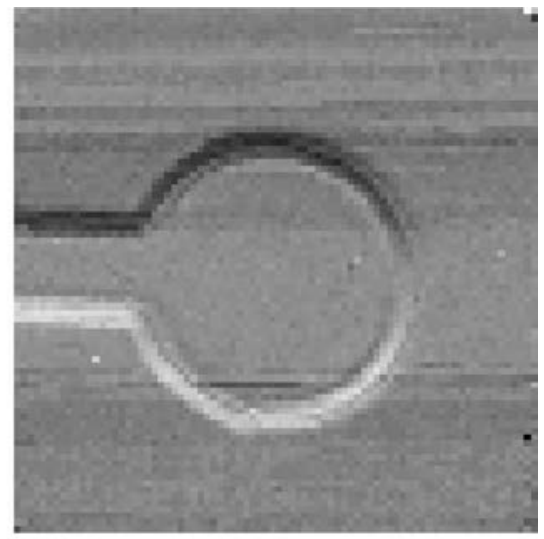

Fig. 5. (a) Initial deformation of the disk surface due to the electrode. (b) Matrix of forces used as source in the model.

with an efficiency proportional to the overlap between their spatial pattern and the source pattern. Because of their abundance, the modes are impossible to distinguish in the temporal behavior of the displacement response. Therefore, we have analyzed the individual experimental modes by taking the $t \rightarrow \omega$ Fourier transform of the signal matrix $S(t, x, y)$, and plotting the $|S(t, x, f)|^{2}$ for discrete eigenfrequencies $\omega$ (Fig. 6). The comparison of the experimental results to the calculated forced modes (Fig. 7) shows a good match for the mode shapes, except for the following striking differences:

- For some modes, there are fairly high differences between the experimental and the predicted frequency.

- Even with the realistic modeling of the source with the help of the 80 by 80 matrix of forces shown in Fig. 5(b), some calculated modes could not be observed experimentally, e.g., the $(0,1)$ mode in the frequency range between 0 and $100 \mathrm{kHz}$. The theoretical modes displayed in Fig. 7 were restricted to those that were observed experimentally.

- When displaying the modes as a function of increasing frequency in a movie, the $(2,1),(4,1),(2,2)$, and $(1,2)$ modes vibration symmetry axis "rotated" in such a way that a symmetry axis for these modes coincided with the horizontal axis after rotation.

The main reason for the discrepancies observed between experimental and theoretical results is most likely the presence of the metal springs used to maintain the disk. These were located at the edge at the angles $0^{\circ}$ and $180^{\circ}$ in Fig. 3 . Although the springs are fairly soft, they still have an ef- 

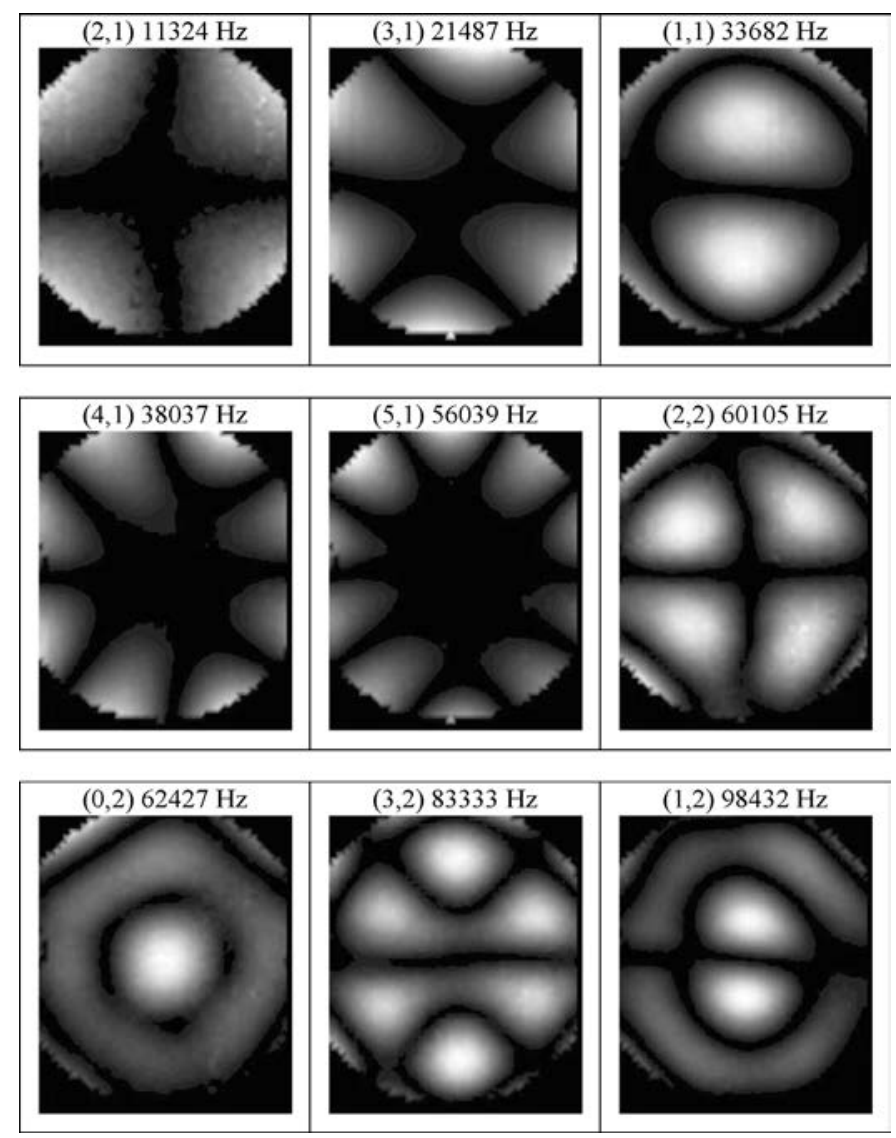

Fig. 6. Experimental results on the forced modes. The disk motion is forced and evolves continuously from mode $(2,1)$ to mode $(1,2)$ from 0 to $100 \mathrm{kHz}$.

fect on the clamping of the disk so that it is not truly free everywhere. The $(0,1)$ mode is such that the displacement of the disk is nonzero everywhere on the edge. Because the two metal springs tend to impose a zero displacement at the clamping points, the $(0,1)$ mode was flattened and did not appear in the experiments.

The modes with a symmetry axis different from the clamping axis seem to have been forced to rotate. It appears that the springs have a non-negligible effect on the resonance frequencies, especially at very low frequency for the $(2,1)$ mode, with an error of about $22 \%$. For higher frequency modes $(3,1),(1,1),(4,1),(5,1),(2,2),(0,2),(3,2)$, and $(1,2)$ the relative error is, respectively, of $5 \%, 0.2 \%$, $6 \%, 1.2 \%, 3.7 \%, 0.1 \%, 0.3 \%$, and $0.2 \%$ and seems to decrease with increasing frequency.

The fact that some theoretical modes have symmetry axes at particular angles [modes $(2,1),(4,1),(2,2)$, and $(1,2)]$ is interpreted as a consequence of the source asymmetry. The source in the model was taken from the experimental results [Fig. 5(b)] and is not perfectly symmetrical. The purely theoretical source made of 40 forces of Section II-C has a symmetry axis as can be seen in the example of Fig. 2. Computing the mode shapes with this source and changing the angle of its symmetry axis shows that the symmetry axes of the calculated modes turn accordingly. The particular angles of the $(2,1),(4,1),(2,2)$,
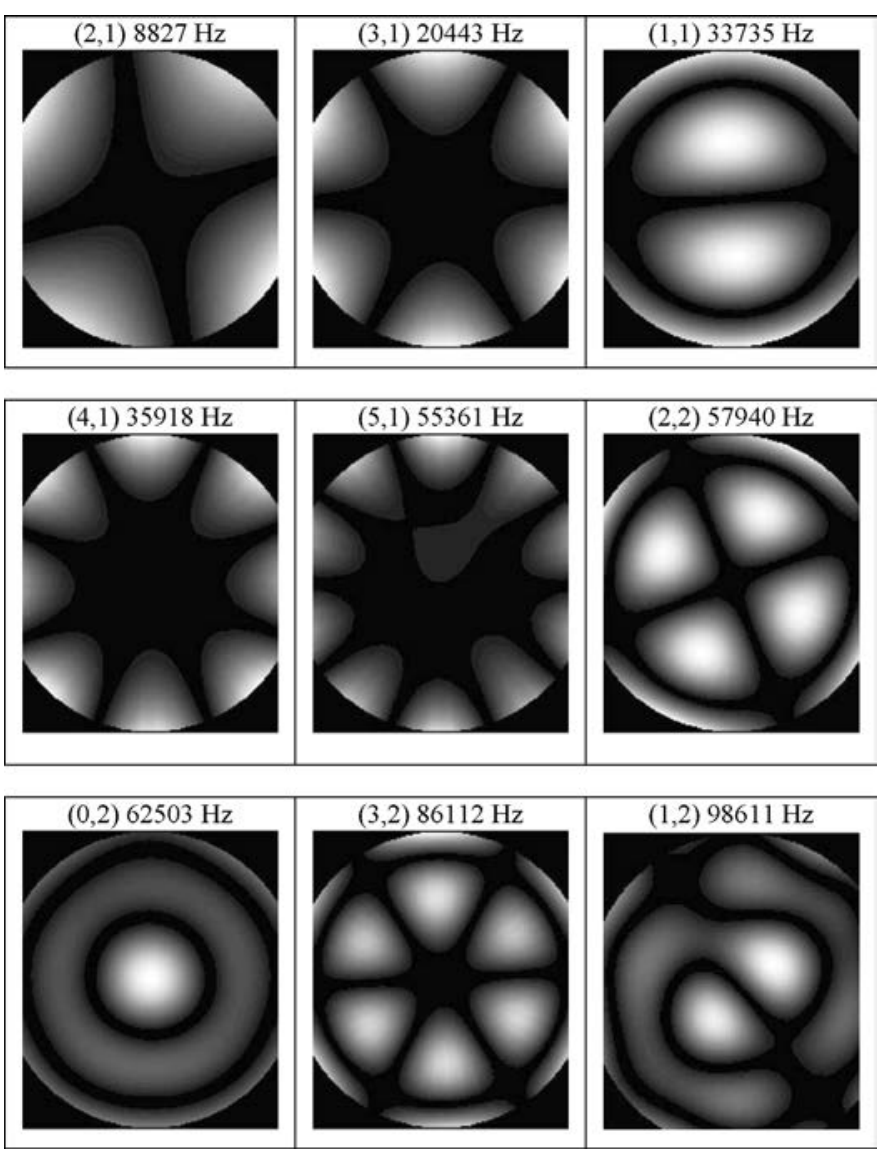

Fig. 7. Theoretical results on the forced modes at frequencies close to those of the normal modes.

and $(1,2)$ modes are determined by the 80 by 80 matrix source and cannot be predicted with precision.

Fig. 8 shows the mean square velocity (proportional to the mechanical energy density [12]) as a function of frequency. As already observed, the clamping springs are responsible for the imperfect match between both the resonance frequencies and the general shape of the curve. The bumpy aspect of the experimental curve at low frequencies is due to the limited time window used for the signal acquisition and to the periodization in the numerical calculation of the Fourier transform. This region of frequencies corresponds to the limit sensitivity of the laser Doppler. The theoretical mean square velocity was calculated in polar coordinates by integrating the values of squared velocity over the disk surface. The $(0,1)$ and the $(6,1)$ modes were not clearly observed experimentally.

\section{Two-Dimensional Wavenumber REPRESENTATION AND COMPARISON WITH LAmb $A_{0}$ Mode}

\section{A. Representation of the Modes in Wavenumber Space}

The top row of Fig. 9 shows the forced bending modes of the disk in the $(x, y)$ plane in a frequency range between $33.3 \mathrm{kHz}$ and $734 \mathrm{kHz}$. Interestingly, the mode shapes at 


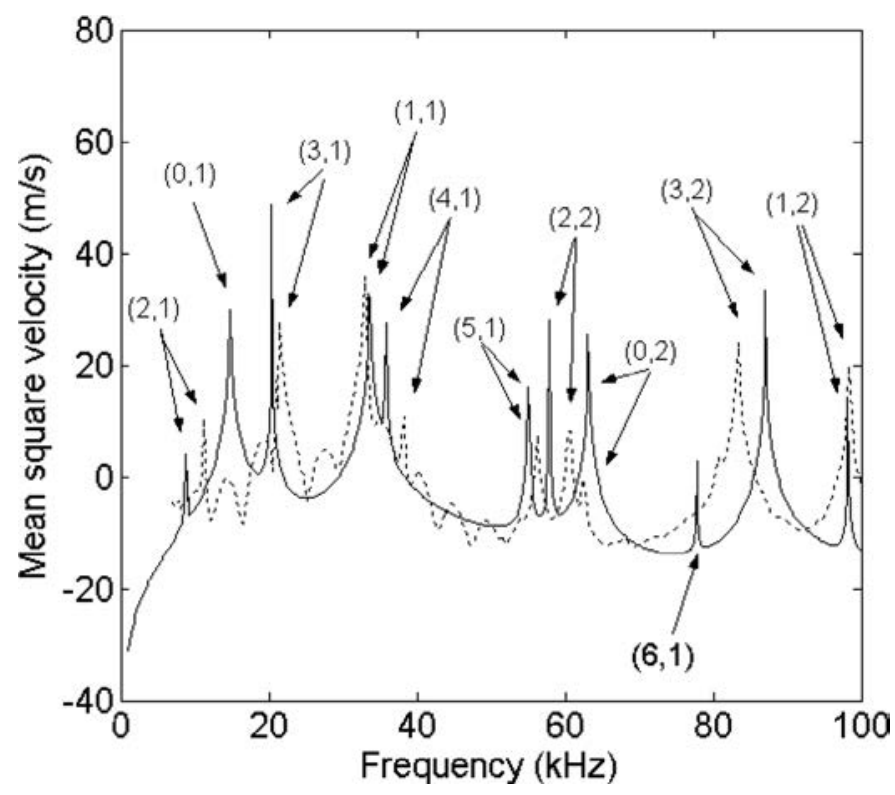

Fig. 8. Mean square velocity as a function of frequency. Plain curve, theoretical; dotted curve, experimental. The $(0,1)$ and the $(6,1)$ modes were not observed experimentally.

$471.1 \mathrm{kHz}$ and $734 \mathrm{kHz}$ are unexpected and not predicted by the theory. An explanation for this occurrance can be that, at higher frequencies, the modes are influenced by the anisotropy of the quartz so that the modes are oriented along certain axes corresponding to the crystallographic axes of the quartz.

The double spatial Fourier transforms (i.e., the $k_{x}$, $k_{y}$ representation) of the experimental forced modes are shown in the middle row of Fig. 9. Modes with complicated shapes in the $x-y$ domain are often simpler in their wavenumber representation. The anisotropy at $471.1 \mathrm{kHz}$ and $734 \mathrm{kHz}$ again appears in the last two images of the middle row. The bottom row of Fig. 9 shows the theoretical wavenumber representation of the forced modes at frequencies close to the experimental frequencies. The theoretical model is valid for an isotropic material, and so the anisotropic behavior neither appears in the last two images of the bottom row nor in the theoretical $x-y$ mode representation. The theoretical results at $98.7 \mathrm{kHz}$ and at $195.6 \mathrm{kHz}$ show once again that the symmetry axes are not horizontal and the experimental ones are, due to the clamping springs. As already observed, the particular angles of the symmetry axis in the theoretical mode shapes at 98.7 and $195.6 \mathrm{kHz}$ are imposed by the source, which is not perfectly symmetrical (the source in the model was an 80 by 80 matrix of forces obtained from experimental results).

\section{B. Experimental and Theoretical Dispersion of the Bending Modes}

The $\left(k_{x}, k_{y}\right)$ representation at different frequencies provides wavenumber-frequency data and yields the dispersion diagram of the bending modes. In Fig. 10, the ex- perimental phase velocities are compared to the velocities obtained theoretically from the model presented in Section II. Each experimental data point was obtained from the angular average of the modulus of the wavevector $\boldsymbol{k}$ at maximum amplitude in the $\left(k_{x}, k_{y}\right)$ plane (Fig. 9, second row) and from using the relation $c_{\text {average }}=\omega / k_{\text {average }}$. The representation of the wavenumber or of the slowness in the $(x, y)$ plane makes it possible to detect the possible anisotropy of the crystal. However, here the anisotropy was averaged out over the angles.

The theoretical data for the phase velocity were obtained from the relation between $\gamma_{n m}$ and $\omega_{n m}$ for the different eigenmodes in (12), which predicts a square root behavior. In our case of a finite disk, the boundary conditions imply the existence of only a discrete number of eigenmodes, resulting in a discrete number of points on the dispersion curve. In Fig. 10, the agreement between the experimental and the theoretical results is fairly good at low frequencies. For frequencies above $40 \mathrm{kHz}$, a systematic difference starts to appear as the frequency increases, to reach a value of about $200 \mathrm{~m} / \mathrm{s}$ at $1 \mathrm{MHz}$. This is due to the limited validity of the approximation in the model of a thin disk. The wavelength at $1 \mathrm{MHz}$ is of about $1.6 \mathrm{~mm}$, which is only 5.3 times greater than the thickness $(0.29 \mathrm{~mm})$. A more accurate dispersion curve for a thicker disk can be obtained from Lamb wave theory in which bending corresponds to the antisymmetrical Lamb $A_{0}$ mode. Its dispersion curve was obtained by numerically solving the Rayleigh-Lamb dispersion equation for guided waves in an isotropic material (see [13] for the derivation of the Rayleigh-Lamb equation). Fig. 10 shows a better agreement between the experimental data and the Lamb $A_{0}$ dispersion curve. At sufficiently low frequencies, the behavior of the Lamb curve corresponds to the square root behavior of the thin disk approximation. Note that, because the bandwidth of the experiment was limited, the wavelengths did not become much smaller than the plate thickness, such that the modes were always guided and the high-frequency Rayleigh surface wave limit of the dispersion curve was not reached.

\section{CONCLusions}

An experimental and theoretical study of bending modes in a partially electroded circular piezoelectric quartz with free edge was proposed. It was found experimentally that the two springs used to maintain the disk in position introduce extra clamping conditions. This resulted in a substantial change in the resonance frequencies for some modes and in a rotation of their symmetry axis toward the axis of the clamping spring.

The classical theory of thin disks was used, and several source shapes were studied. A collection of forces obtained from the experimental results was used to model a realistic source to simulate the experimental condition. The model only describes bending modes and does not include thickness shear or compressional modes. It does not account 

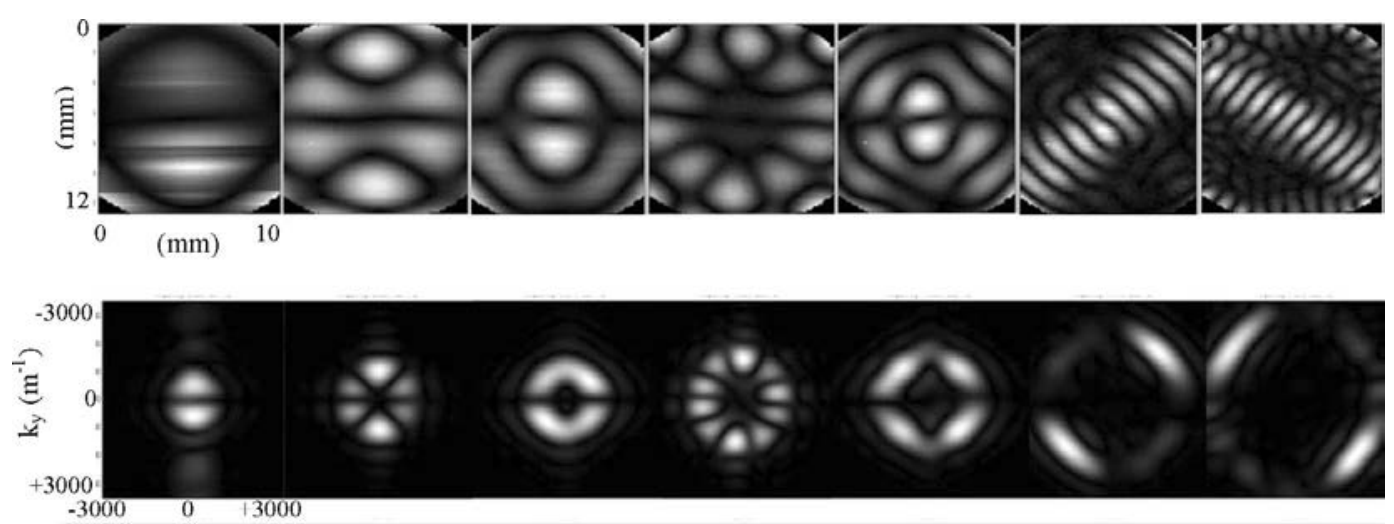

$\left(\mathrm{k}_{\mathrm{x}}\left(\mathrm{m}^{-1}\right)\right.$
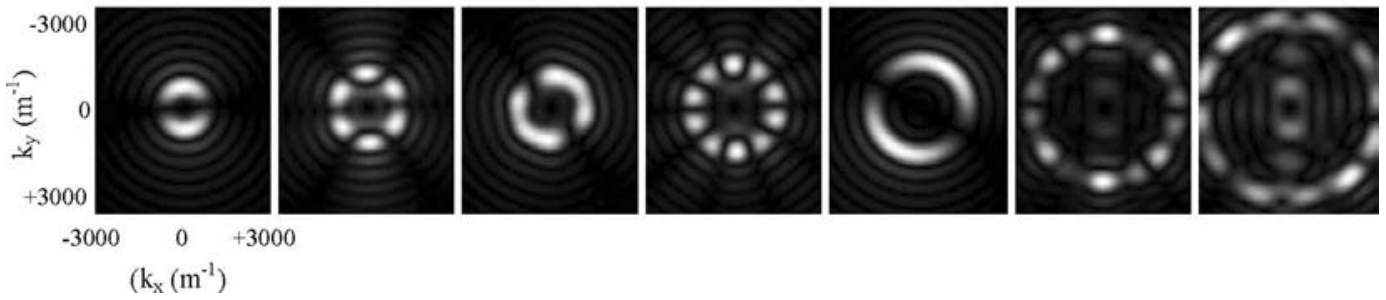

Fig. 9. Top row: spatial $(x, y)$ dependence of the displacement amplitude of bending eigenmodes. From left to right: deformation amplitude at $33.3 \mathrm{kHz}, 83.3 \mathrm{kHz}, 98.7 \mathrm{kHz} 152.6 \mathrm{kHz}, 195.2 \mathrm{kHz}, 471.1 \mathrm{kHz}$, and $734 \mathrm{kHz}$. Middle row: Amplitude $\left(k_{x}, k_{y}\right)$ representation of the experimental disk deformation at the same frequencies. Bottom row: theoretical modes at $33.2 \mathrm{kHz}, 82.9 \mathrm{kHz}, 98.5 \mathrm{kHz}, 157.5 \mathrm{kHz}, 195.6 \mathrm{kHz}$, $472 \mathrm{kHz}$, and $733.9 \mathrm{kHz}$. The units are shown in the first image of each row.

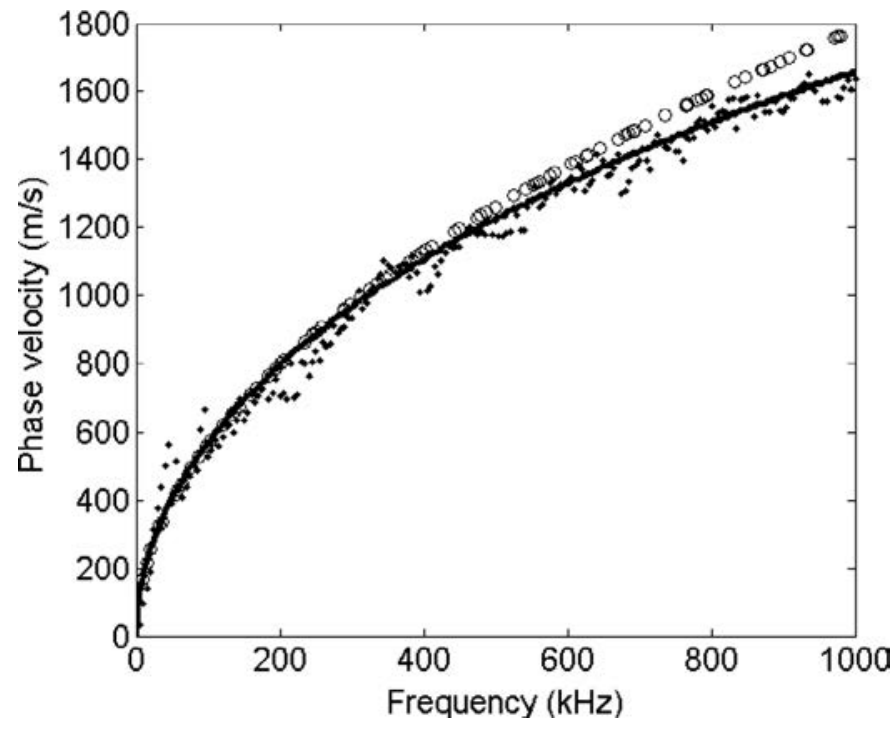

Fig. 10. Comparison between the experimental phase velocities (black dots) obtained from the angular average of the maximum values of $\boldsymbol{k}$ for every frequency via the relation $c=\omega / k$ in the diagrams of Fig. 9 (middle row) and the dispersion curve obtained from the classical theory of plates and disks presented in Section II (circles), and the first antisymmetrical $A_{0}$ Lamb mode (plain curve).

for the electromechanical coupling, i.e., the stiffening effect. However, the agreement between experimental and theoretical results on the mode shapes using this source was fairly good.

A wavenumber analysis of the experimental modes showed the presence of anisotropy related to the crystallographic axes at higher bending frequencies. However, the anisotropy was weak and, although the theoretical model was developed for isotropic materials, the experimental and theoretical results corresponded quite well. There was only a slight discrepancy between the dispersion curves at high frequencies, at which the thin disk approximation of the model leads to a deviation from the Lamb $A_{0}$ mode, which matches very well the experimental data in the whole accessible frequency range.

\section{REFERENCES}

[1] E. Benes, M. Gröschl, W. Burger, and M. Schmid, "Sensors based on piezoelectric resonators," Sens. Actuators A, vol. 48, pp. 1-21, 1995.

[2] C. E. Reed, K. K. Kanazawa, and J. H. Kaufmann, "Physical description of a viscoelastic loaded AT-cut quartz resonator," $J$. Appl. Phys., vol. 68, pp. 1993-2001, 1990.

[3] H. F. Tiersten, "Thickness vibrations of piezoelectric plates," $J$. Acoust. Soc. Amer., vol. 35, pp. 53-58, 1963.

[4] B. K. Sinha and D. S. Stevens, "Thickness-shear vibrations of a beveled AT-cut quartz plate," J. Acoust. Soc. Amer., vol. 66, pp. 192-196, 1979.

[5] J. Détaint, J. Schwartzel, C. Joly, and E. Philippot, "Energy trapping in plane and corrugated resonators: Application to quartz and Berlinite," in Proc. 41st Annu. Freq. Contr. Symp., 1987, pp. 314-324.

[6] L. Martinez, E. Caplain, S. Serfaty, P. Griesmar, G. Gouedard, and M. Gindre, "Chirp-Z analysis for sol-gel transition monitoring," Ultrasonics, vol. 42, pp. 507-510, 2004.

[7] C. H. Huang, Y. C. Liu, and C. C. Ma, "Theoretical analysis and experimental measurement for resonant vibration of piezoceramic plates," IEEE Trans. Ultrason., Ferroelect., Freq. Contr., vol. 51, pp. 12-24, 2004.

[8] C. H. Huang, "Theoretical and experimental vibration analysis for a piezoceramic disk partially covered with electrodes," $J$. Acoust. Soc. Amer., vol. 118, pp. 751-761, 2005. 
[9] P. M. Morse and K. U. Ingard, Theoretical Acoustics. Princeton, NJ: McGraw-Hill, 1968, pp. 213-222.

[10] T. B. Gabrielson, "Frequency constants for transverse vibration of annular disks," J. Acoust. Soc. Amer., vol. 105, pp. 3311$3317,1999$.

[11] S. M. Vogel and D. W. Skinner, "Natural frequencies of transversely vibrating uniform annular plates," J. Appl. Mech., vol. 32, pp. 926-931, 1965.
[12] O. Foin, J. Nicolas, and N. Atalla, "An efficient tool for predicting the structural acoustic and vibration response of sandwich plates in light or heavy fluid," Appl. Acoust., vol. 57, pp. 213242, 1999.

[13] D. Royer and E. Dieulesaint, Elastic Waves in Solids: Free and Guided Propagation. vol. 1, Berlin: Springer Verlag, 1999. 